Rakenteiden Mekaniikka (Journal of Structural Mechanics)

Vol. 50, No 3, 2017, pp. $162-165$

https://rakenteidenmekaniikka.journal.fi/index

https://doi.org/10.23998/rm.64953

(c) The author(s) 2017.

Open access under CC BY-SA 4.0 license.

\title{
Shell finite elements, with emphasis on strategies used within Elmer software
}

\author{
Mika Malinen
}

Summary. The best way to handle finite element discretization of structural shell equations is still a subject of discussion. This article presents some viewpoints and reviews innovations which have recently been taken into use in connection with the implementation of new shell finite elements into the open source finite element software Elmer.

Key words: shell, finite element, lines of curvature coordinate

Received 16 June 2017. Accepted 24 July 2017. Published online 21 August 2017

\section{Introduction}

Paradoxically, not much of the general equations for structural shells as formulated in the classical shell theory [3] is nowadays utilized directly by common software packages that employ the finite element method. The complicated shell equations written by using tensor notation were perhaps beyond the grasp of many engineers and simpler concepts were adopted by the engineering community in the 1970s. The idea of degenerated solid elements was then taken into use and common finite elements are still based on this concept [1], although some new notable ideas related to isogeometric analysis have also emerged more recently.

While use of classical shell models could have been an option for early engineers, it cannot be denied that using such a model as a starting point is not indeed straightforward when the goal is to achieve a generic finite element procedure which can handle general shells without unnecessary constraints on the geometry of the mid-surface of the shell. The classical shell theory is built on a given global parametrization of the mid-surface of the shell, so that the reference configuration of the shell body can be described in terms of a system of normal coordinates. However, such a global parametrization is not usually available directly when a computational model is generated by using a geometry representation available within computer-aided design (CAD) system. Nevertheless, this is not an issue in connection with the degenerated solid approach where the coordinates of a reference finite element give an adequate local parametrization of part of an approximate shell body.

At the same time, it must also be noted that the complexity of the classical shell 
equations could be reduced significantly by choosing a clever parametrization for the mid-surface of the shell so that the system of normal coordinates could be associated with an orthogonal or even orthonormal basis. Indeed, such a choice was frequently considered in the classical shell theory by restricting consideration to use of lines of curvature coordinates, but again ready representations of surfaces in this form are not generally available in practice. Actually, devising a practical method for reparametrizing a given surface globally in terms of lines of curvature coordinates is a nontrivial problem per se, and lines of curvature coordinates have not gained much attention in recent literature on shells.

We conclude that a lack of global surface parametrizations seems to support even today the idea that in connection with finite elements the geometry of the mid-surface is best described by using an elementwise parametrization. On the other hand, the standard geometry description of the degenerated solid approach, which is based on independent equal-order Lagrange interpolation of the mid-surface position and the director vector, leads to discrete models whose mathematical analysis has proved to be more complicated than the analysis of discretizations derived from the classical shell equations posed over a two-dimensional domain. A lack of true normal coordinates leads to favouring an interpretation where the discrete model is considered to approximate a three-dimensional variational problem; cf. [1]. While there is of course nothing objectionable in the threedimensional interpretation, there is a risk that some understanding springing from the study of conventional two-dimensional theories may be shaded at the same time. For example, the asymptotic behaviour of shell models has traditionally been studied in connection with two-dimensional theories. In modern perspective, this knowledge is essential in understanding constraints that relate to the asymptotic behaviour of shell deformations and, hence, in designing nonstandard variational formulations which enable finite elements that are less prone to locking effects [1].

If a developer of finite element software was given a free hand in choosing a method to be implemented for structural shell analysis, we would now pose the question of what strategy should then be followed. This question was indeed met by the author of the present paper recently when the objective was to renew the shell model of the open source finite element software Elmer [2]. An outcome of this consideration has been an alternate finite element solver that combines ingredients from both the classical shell theory and use of the standard finite element data about the shell geometry. It is built on gradual developments put forward in [4], [5] and, to some extent, already in [6] without a practical implementation. The aim of the present paper is to summarize the key ideas that are now utilized by the shell solver that is available within Elmer.

\section{Shell finite elements as implemented in Elmer: The key ideas}

The approximation of the geometry of the shell is usually based on data which give the position of the mid-surface of the shell and the shell director at the nodes of finite elements. In the first place special forms of solid elements are usually created by using equal-order Lagrange interpolation of the coordinates of mid-surface position and the director vector.

In [4] we have however introduced an alternate way to reconstruct the geometry of the shell from the same data which is conventionally employed in connection with the degenerated solid approach. The key idea is to create first a collection of space curves which give improved approximations of curved element edges. This is basically achieved by applying the Hermite interpolation so that the edge curve goes through the edge nodes 
and its tangent vector is perpendicular to the director vector given at the nodes. It is notable that even the lowest-order geometry data leads to a relatively high accuracy as we may expect fourth-order accurate approximation with respect to an edge size parameter in $L^{2}$ (for the definition of space $L^{2}$ see [1]). The technique of finite element blending (see [7], where this technique is approached via the concept of transfinite interpolation) is next applied in order to get an improved mid-surface approximation which is consistent with the improved approximations of curved element edges obtained before. As discussed in [4], it is possible to generate fourth-order accurate approximation also for the mid-surface position by additionally considering a quadrilateral element as a macro element for a subtriangulation. This strategy can also be chosen with Elmer in order to avoid using simpler and possibly less accurate approximation based on serendipity finite elements.

In the classical shell theory all geometric model parameters are basically derived via differentiation of the mapping (chart) which defines the position of the shell mid-surface in its reference configuration. For example, the second fundamental form of the surface, which is related to curvature, depends on the second-order partial derivatives of the chart. It should be noted that the curvature information could not be reproduced in a straightforward and consistent manner from the approximate chart based on the lowest-order Lagrange interpolation of the coordinates of mid-surface position. However, the improved surface approximation which is now available within Elmer can be used to yield elementwise estimates of all geometry parameters needed in the finite element discretization of conventional shell equations. It is also notable that the tangent plane to the approximate surface is uniquely defined at each node and agrees with the initial data.

Proceeding from the improved surface approximation, an elementwise Monge patch can then be created. That is, the coordinates of points of the local surface with respect to a local Cartesian coordinate frame can be considered to be obtained in a form $\left(x^{1}, x^{2}, z\left(x^{1}, x^{2}\right)\right)$ where $z$ is a finite element function of two variables. More specifically, the origin of the local coordinate system associated with the Monge patch is chosen to be the position of the approximate mid-surface evaluated for the parameter values corresponding to the midpoint of the reference element. An eigenvalue problem in terms of the second fundamental form of the approximate mid-surface is also solved in order to align the local coordinate system such that two of its orthonormal basis vectors give the orientation of lines of curvature coordinate curves at the origin. This brings us to the simplest approximation of a parametrization corresponding to lines of curvature coordinates. A refined approximation can also be created with Elmer such that it is consistent with the fourth-order accurate estimation of the mid-surface position; cf. [5]. This process corresponds to finding a reparametrization of the local surface so that the points of the local surface are obtained in a form $\left(x\left(y^{1}, y^{2}\right), y\left(y^{1}, y^{2}\right), z\left(x\left(y^{1}, y^{2}\right), y\left(y^{1}, y^{2}\right)\right)\right)$ where $x: K \rightarrow \mathbb{R}$ and $y: K \rightarrow \mathbb{R}$, with $K \subset \mathbb{R}^{2}$.

Finally, the problem of approximating the potential energy of the shell can be considered. Since we can use a true system of normal coordinates and the basis associated with lines of curvature coordinates is naturally orthogonal, the process implemented into Elmer follows closely that of the classical shell theory, although here the surface parametrization is local rather than global. Refining the shell model as worked out already in [6] using lines of curvature coordinates becomes also an option for creating finite elements that can approximate three-dimensional effects in a more faithful manner and can also be coupled with solid finite elements. This refinement also simplifies the definition of compatibility relations between local finite element approximations. 


\section{References}

[1] D. Chapelle, K.J. Bathe. The Finite Element Analysis of Shells - Fundamentals, 2nd Edition. Springer, 2011.

[2] Elmer finite element software homepage. http://www.csc.fi/elmer.

[3] P.M. Naghdi. Foundations of elastic shell theory. In Progress in Solid Mechanics, I.N. Sneddon, R. Hill (Eds), Vol. 4, North-Holland, 1-90, 1963.

[4] M. Malinen. Improved surface reconstruction from conventional geometry data for general shell finite elements. In Proceedings of the 29th Nordic Seminar on Computational Mechanics - NSCM29, R. Larsson (Eds), Chalmers University of Technology, Göteborg, 2016. http://publications.lib.chalmers.se/records/fulltext/ 244480/_ocal_244480.zip.

[5] M. Malinen. Generating lines of curvature coordinates for finite element modelling. In Proceedings of the XII Finnish Mechanics Days, R. Kouhia, J. Mäkinen, S. Pajunen, T. Saksala (Eds), Finnish Association for Structural Mechanics, 106-111, 2015. http: //rmseura.tkk.fi/smp_proceedings/SMP_2015_Proceedings.pdf

[6] M. Malinen. Finite elements and linear shell theory - Studies on shell models and numerical locking phenomena. Doctoral thesis, Helsinki University of Technology, Publications in Mechanics of Materials, TKK-LO-34, 2001.

[7] P. S̆olín, K. Segeth and I. Doležel. Higher-Order Finite Element Methods. Chapman \& Hall/CRC, 2004.

Mika Malinen

CSC - IT Center for Science

P.O. Box 405

FI-02101 Espoo

Finland

mika.malinen@csc.fi 